\title{
Cubic and hexagonal InGaAsN dilute arsenides by unintentional homogeneous incorporation of As into InGaN
}

\author{
F.M. Morales, D. Carvalho, T. Ben, R. García, S.I. Molina, A. Martí, \\ A. Luque, C.R. Staddon, R.P. Campion and C.T. Foxon
}

\begin{abstract}
Arsenic alloying is observed for epitaxial layers nominally intended to be $\operatorname{In}_{0.75} \mathrm{Ga}_{0.25} \mathrm{~N}$. Voids form beneath their interfaces with $\mathrm{GaAs}$ substrates, acting as sources of $\mathrm{Ga}+\mathrm{As}$ out-diffusion into the growing epilayers. As a result, heteroepitaxial single-phase quaternary $\operatorname{In}_{x} \mathrm{Ga}_{1-x} \mathrm{As}_{y} \mathrm{~N}_{1-y}$ films are formed with $x \sim 0.55$ and $0.05<y<0.10$. While an undoped epilayer retains the wurtzite structure, a Mn-doped sample showed randomly spaced dopant segregations, which, together with a slightly higher As concentration, led to a transformation from the hexagonal to the twinned cubic phase.
\end{abstract}

Keywords: Compound semiconductors (InGaAsN); Dilute arsenide; Transmission electron microscopy (TEM); X-ray diffraction (XRD)

The (In)GaAsN/GaAs semiconductor system has been raised as a potential industrial solution due to the surprising optical and electronic properties attained by the incorporation of small amounts of nitrogen into the (In) GaAs lattice [1]. The As-rich end point of the $\mathrm{In}_{x} \mathrm{Ga}_{1-x} \mathrm{As}_{y} \mathrm{~N}_{1-y}$ quaternary alloy is known as a dilute nitride, in contrast to the conventional III-N nitrides having $50 \%$ of N. Similarly, large modifications of the band structure have been anticipated near the N-rich point of GaAsN since small concentrations of As cause the electronic properties of the ternary alloy to deviate drastically from those predicted by linear interpolations using the virtual crystal approximation [2]. This dilutearsenide concept, or even alloys with intermediate As- $\mathrm{N}$ concentrations, has not still been technologically exploited. Recently the $\mathrm{GaAs}_{y} \mathrm{~N}_{1-y}$ ternary has been fabricated for the whole compositional range [3]. The crystal structures destabilized for values $0.17<y<0.75$. However, even though reaching this onset of amorphization the films presented a smooth morphology, homogeneous compositions and sharp, well-defined optical absorption edges in different crystalline and amorphous substrates [4].
On the other hand, the fabrication of low-In content InGaN is well-controlled for optoelectronics [5] but there have been great efforts to achieve good-quality homogeneous alloys at the medium and high In concentrations [6] to access longer wavelengths. However, to achieve this one must combat against the compositional or structural phase separations common at these ranges [7,8]. Additionally, for the chance to tune the wurtzite structure of III-Ns to zinc blende, one provides extra advantages such as more isotropic properties and alternative band gap for the cubic phase [9]. In this work we describe the synthesis of hexagonal and cubic single-phase $\mathrm{In}_{x} \mathrm{Ga}_{1-x} \mathrm{As}_{y} \mathrm{~N}_{1-y}$ films with $x \approx 0.55$ and $0.05<y<0.10$ on GaAs substrates. These alloys with unprecedented compositions may provide a combination of benefits as a consequence of their intermediate-In composition and low-As content.

A control layer (sample A) was deposited by plasmaassisted molecular beam epitaxy, on Si-doped (111)B GaAs, in a home-made MBE machine with an ultimate base pressure of $10^{-9}$ mbar. The substrate was heated to $620^{\circ} \mathrm{C}$ in order to remove the native oxide and then nitrided at $\sim 650^{\circ} \mathrm{C}$ using a nitrogen plasma CARS25 RF source. The temperature was later reduced to $\sim 500^{\circ} \mathrm{C}$ to start the growth of InGaN using conventional elemental sources for $\mathrm{Ga}$ and In. More detail on the 
growth method can be found elsewhere [10]. In a second specimen (sample B) the same procedure is repeated until a thin InGaN layer is grown $(\sim 20 \mathrm{~nm})$ before tuning on the Mn flux at a level intended to get a doping level of $1 \%$ in the InGaN layer. Growth takes place close to stoichiometry, and the conditions were adjusted with the aim to get $\mathrm{In}_{0.75} \mathrm{Ga}_{0.25} \mathrm{~N}$ layers in both cases.

$\mathrm{X}$-ray diffraction (XRD) studies were performed using a Philips X'Pert MRD system and $2 \theta / \omega$ linescans were measured. The samples were also prepared for transmission electron microscopy (TEM) in cross-sections by mechanical thinning plus ion milling. The interplanar spacings of the epilayers were measured by selected area electron diffraction (SAED) in a Jeol-1200-EX transmission electron microscope. High-resolution TEM (HRTEM) micrographs and high-angle annular dark field (HAADF) images were taken in a Jeol-2010F TEM microscope, where studies of compositions were carried out by energy dispersive X-ray (EDX) and electron energy-loss (EELS) spectroscopies in scanning TEM mode (STEM).

Quantitative analyses of the In/Ga relative contents in the epilayers by EDX, calibrated following steps reported elsewhere [6], indicated ratios of 56/44 and 57/ 43 for samples A and B, respectively. The standard deviations of the averaged values of proportional weights of the present elements, made by tens of nano-beam analyses along and across every layer, did not exceed the nominal value of $2 \%$ of maximum absolute error for this technique. Moreover, a small presence of As in both layers could not be ruled out attending to the EDX signals associated with arsenic that qualitatively showed a slightly higher content in sample B.

HRTEM micrographs show that the obtained top layers are heteroepitaxial and sharply interfaced to the GaAs. However, while the formed InGaAsN epilayer is hexagonal $(h)$ and single-phase (lattice coherent with well-coalesced domains without compositional phase separation), the use of $\mathrm{Mn}$ promoted the appearance of the InGaAsN:Mn twinned cubic (c) polytype as the prevailing phase after an $h$-type portion was initially formed. The observed orientation relationships, being the notation $\mathrm{G}=\mathrm{GaAs}, \mathrm{H}=h$-InGaAsN, $\mathrm{H} 2=h$-InGaAsN:Mn, and $\mathrm{C}=c$-InGaAsN:Mn, are $[0001] \mathrm{H} /\left[\begin{array}{lll}1 & 1 & 1\end{array}\right] \mathrm{G} ;\left[\begin{array}{lll}1 & 1 & 2\end{array}\right] \mathrm{H} /$

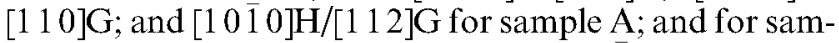

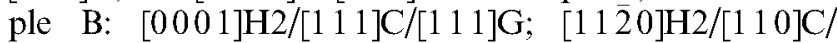

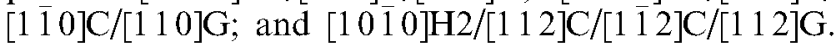
This conclusion is also extracted from diffraction analyses (Fig. 1).

By using a novel method of high-resolution electron diffraction [11] the $c$-lattice parameters of phases $\mathrm{H}$ and $\mathrm{H} 2$ were measured to be $5.5472 \AA$ and $5.5690 \AA$, respectively. These measurements are in agreement with XRD values and rely on the fact that the reflections associated to unstrained $3 \mathrm{C}-\mathrm{GaAs}$ constitutes an internal standard to measure relative distances in SAED patterns. Note that both the (0004)-H2 and (222)-C reflections are coincident as expected for two different behaving $2 \mathrm{H}-$ and $3 \mathrm{C}$-polytypes of the same composition with (0001) and (111) habit planes, respectively. Therefore, considering that both layers are closedpacked and strain-relaxed, by using the relationship $a_{0}{ }^{3 \mathrm{C}-\mathrm{InGaAsN}}=\sqrt{3} / 2 \cdot c_{0}{ }^{2 \mathrm{H}-\mathrm{InGaAsN}}[12]$, the a-lattice constant of the cubic phase in sample B is calculated to be $4.8229 \AA$.

It is possible, then, to calculate the $\mathrm{As} / \mathrm{N}$ ratio in an homogeneous cubic or hexagonal $\operatorname{In}_{x} \mathrm{Ga}_{1-x} \mathrm{As}_{y} \mathrm{~N}_{1-y}$ alloy by considering the previous relationship and applying Vegard's law; in particular, by the use of the following linear interpolation:

$$
\begin{aligned}
d_{0}(x, y)= & x \cdot y \cdot d_{0}^{I n A s}+x \cdot(1-y) \cdot d_{0}^{I n N}+y \cdot(1-x) \\
& \cdot d_{0}^{G a A s}+(1-y) \cdot(1-x) \cdot d_{0}^{G a N}
\end{aligned}
$$

Given that $x$ is already known, $y$ can be solved considering the binary constants $a_{0}$ GaAs $=5.65325 \AA$ and $a_{0}{ }^{\operatorname{InAs}}=6.0583 \AA$ for the arsenides [13] and $c_{0}{ }^{\operatorname{InN}}=$ $5.70374 \AA$ and $c_{0}{ }^{G a N}=5.1850 \AA$ for the nitrides [6]. Hence, in sample $A$ the solution is $y=0.05$ while in sample $\mathrm{B}$ it is $y=0.07$. Note that these results are in agreement with the trend of the EDX observations of As contents and that similar solutions were reached when using bibliography data of wurtzite InAs and $\mathrm{GaAs}$ and of zine blende $\mathrm{InN}$ and $\mathrm{GaN}$ for comparisons. Moreover, if $\operatorname{In}_{x} \mathrm{Ga}_{1-x} \mathrm{~N}$ layers were considered as the only possibility, the solutions of $x$ by applying Vegard's rule would be 0.70 and 0.74 in samples A and B, respectively. These results are evidently far away from those directly measured by EDX and are an indication of the homogeneous incorporation of As.

Two main questions arise from the results shown. One of them is to answer how As atoms have been incorporated into the epilayers without arsenic flux being input as an external source. The second is to explain the difference between both samples from the point of view of the distinct crystal phases formed. Considering the thicknesses and growth times for both InGaAsN films ( $240 \mathrm{~nm}$ in sample A and $300 \mathrm{~nm}$ in sample B) it can be concluded that the growth rate was approximately the same $\left(\sim 150 \mathrm{~nm} \mathrm{~h}^{-1}\right)$. The roughness in both samples is also similar and reached $25-50 \mathrm{~nm}$. Therefore superficially there are no gross differences between the samples. However, distinguishable extended structural defects were found and characterized as threading dislocations (TDs), stacking faults (SFs), twins, domain boundaries, or empty and material-filled voids at the GaAs substrate below the interface. Figure 2 shows some of these features. In this way, the hexagonal film (sample A) is formed by a coalesced columnar structure where the domains are $\sim 100-150 \mathrm{~nm}$. When displaying the structures in diffraction-contrast mode ( $g=0002$ of the nitride), TDs are visible, as expected for medium-/high-quality wurtzite single-crystalline nitrides. The density of these TDs decreases as the thickness of InGaAsN increases. In the case of the cubic film (sample B), two twinned variants were often found on top of the InGaAsN hexagonal layer previously formed.

The voids found in the GaAs just below their interfaces with InGaAsN are partially or completely empty, being faceted in the high-density cubic planes as inverted tetrahedral shapes, sometimes being truncated. Our hypothesis is that Ga and As out-diffusion played some role during the growth. Indeed, the $\mathrm{Ga}$ and As relative contents have increased from the expected, as presented, in comparison with the originally intended $\operatorname{In}_{0.75} \mathrm{Ga}_{0.25} \mathrm{~N}$ composition. These voids, typically observed in other 

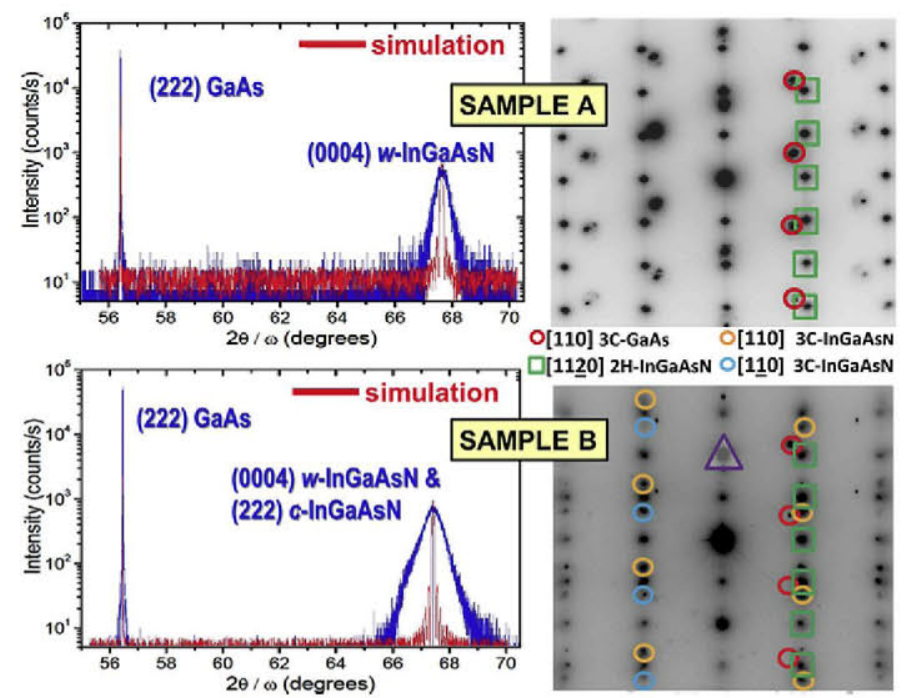

Figure 1. XRD scans and electron diffraction patterns with indexed reflections registered in the [110] zone axis of the GaAs. The triangle at the bottom SAED pattern indicates the coincident position of InGaAsN:Mn hexagonal (0004) and cubic \{222\} diffraction spots.

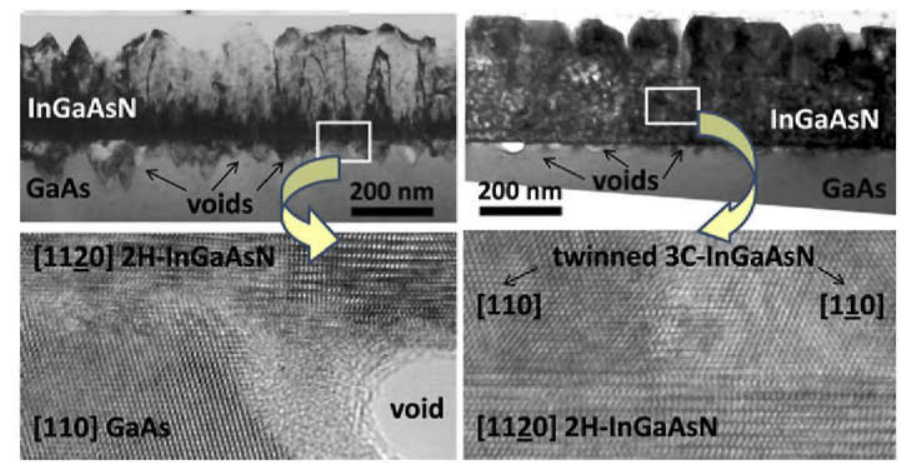

Figure 2. Panoramic TEM micrographs and details of samples A (left) and B (right). The HRTEM insets show the $[11 \overline{2} 0] \mathrm{H} /[110] \mathrm{G}$ and the

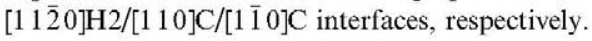

systems (as in $\mathrm{SiC} / \mathrm{Si}[14]$ ), can be expected for a heterostructure where atomic species of the substrate may participate on the formation of a growing top layer. In similar structures to the ones presented here, this type of surface pitting behaviour has been observed in $\mathrm{MBE}$ growth of GaN on GaAs or GaP [15].

On the other hand, for sample $\mathrm{B}$ the role of $\mathrm{Mn}$ should not be ignored. Figure 3 shows an example where Mn was found not to be homogeneously distributed, but segregated in regions above the substrate. This might be one of the reasons why the hexagonal layer turned to cubic after the $\mathrm{Mn}$ introduction since these accumulations can alter the growth mechanism, promoting points of nucleation of planar defects in the previously smooth growing front. For example, $\mathrm{Mn}$ doping of $\mathrm{ZnSe}$ on GaAs can form different phases which act as heterogeneously distributed sources of SFs [16]. The Mn L-edge map extracted from the EELS spectrum image corresponding to the inset of the HAADF-STEM micrograph shows one of these points of preferential segregation (the inset has $50 \mathrm{~nm}$ width and the $\mathrm{Mn}$ rich area is displayed by yellow pixels).

In this way, the onset of twins and SFs were found just at the places of transitions between the hexagonal ( $\mathrm{ABAB} . .$. stacking sequence of the closed packed layers)

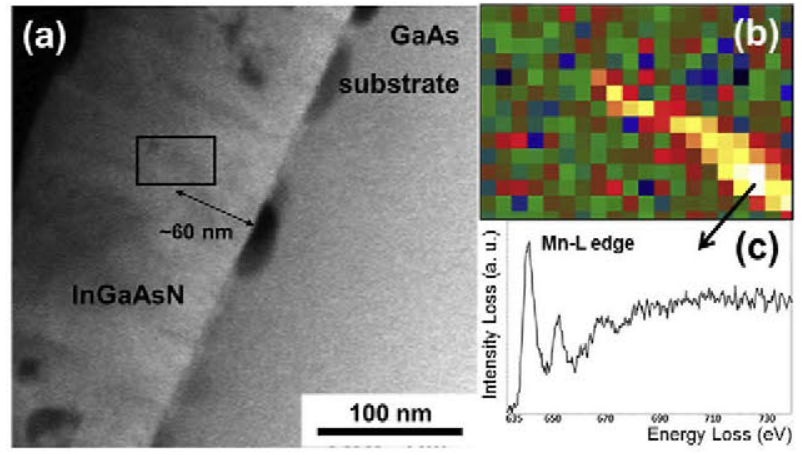

Figure 3. (a) HAADF overview of sample B; (b) EELS elemental map of the Mn signal extracted from the squared region in the image presented in (a) where a higher Mn content is indicated in yellow; (c) individual spectrum for the $\mathrm{Mn}-\mathrm{L}$ edge. (For interpretation of the references to colour in this figure legend, the reader is referred to the web version of this article.)

and cubic ( $\mathrm{ABCABC} . .$.$) phases. In this way, these re-$ gions of phase switching were observed to be placed $\sim 50 \mathrm{~nm}$ above the GaAs/InGaAsN interface (see Fig. 4).

On a hexagonally arranged (111) surface like that used in this work it is expected that the lower energy 


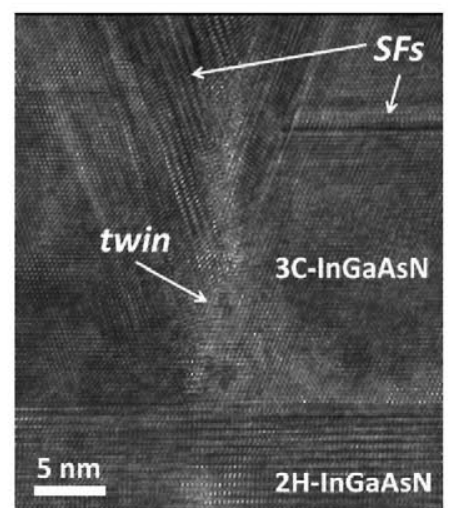

Figure 4. HRTEM micrograph highlighting planar defects formed after the onset of the hexagonal to cubic InGaAsN transformation.

wurtzite phase of InGaN will be formed, as it happened in the initial layer of sample B unaffected by Mn. However, the strain energy accumulated by the appearance of planar defects (stacking disorder) can assist the transformation between two polytypes with a small difference of enthalpies of formation. The stress may lead to a stabilization of the cubic phase, because plastic deformation in hexagonal polytypes initiates a transformation into the pure cubic 3C-polytype [17]. The fact that there is As in the layer can also enhance the cubic phase to be formed (cubic GaAs is the lower energy form of this compound). It is well known that a deliberate use of As flux as a surfactant can encourage the cubic phase of $\mathrm{GaN}$ to be produced [18] and can reduce the formation of voids if grown on GaAs. This way of having cubic seeds, combined with the further removal of the substrate, is nowadays the basis of the standard method of fabrication of free-standing GaN, $\mathrm{AlN}$ and $\mathrm{AlGaN}$ substrates of the zinc blende type [19].

The unintentional homogeneous incorporation of arsenic is shown for layers deposited by molecular beam epitaxy, nominally intended to be $\operatorname{In}_{x} \mathrm{Ga}_{1-x} \mathrm{~N}$ with $x=0.75$. In this way, single-crystalline dilute arsenide (high- $\mathrm{N}$ content) layers of $\sim 250-300 \mathrm{~nm}$ are heteroepitaxially deposited on (111) GaAs substrates having compositions of $\mathrm{In}_{0.56} \mathrm{Ga}_{0.44} \mathrm{As}_{0.05} \mathrm{~N}_{0.95}$ and $\mathrm{In}_{0.57} \mathrm{Ga}_{0.43} \mathrm{As}_{0.07} \mathrm{~N}_{0.93}$. Just beneath the InGaAsN/GaAs interfaces, big voids were formed which appear to act as a source for the atomic out-diffusion of $\mathrm{Ga}$ and As into the growing epilayer. The use of $\mathrm{Mn}$ incorporation at a doping level $(\sim 1 \%)$ has also been tested. While an undoped layer retains the wurtzite structure having an orientation relationship of [1 1120$]$ 2H-InGaAsN/[1 10$]$ GaAs; the doping growth promoted randomly spaced $\mathrm{Mn}$ segregations, which together with a slightly higher As concentration, led to a sudden transformation from the hexagonal to the twinned cubic phase having the epitaxy of $[110] 3 \mathrm{C}$ InGaAsN/[1 10$] \mathrm{GaAs}$.
These facts were proved by X-ray diffraction and transmission electron microscopy techniques. The achieved compositions for these quaternary alloys were not previously reported and are of great technological interest.

This work was supported by the Junta de Andalucía (PAI group TEP-120 and projects P09TEP-5403 and P08-TEP-03516) and by the CICYT project MAT 2010-15206. UE-FEDER co-financing to Junta de Andalucía projects is also acknowledged. EU project IBPOWER (ref. 211640) and EU COST MP0805 Action are also to be addressed. TEM analyses were carried out at DME, SCCYT-UCA.

[1] M. Henini, Dilute Nitride Semiconductors, Elsevier, Amsterdam, 2005.

[2] J. Wu, W. Walukiewicz, K.M. Yu, J.D. Denlinger, W. Shan, J.W. Ager III, A. Kimura, H.F. Tang, T.F. Kuech, Phys. Rev. B 70 (2004) 115214.

[3] K.M. Yu, S.V. Novikov, R. Broesler, I.N. Demchenko, J.D. Denlinger, Z. Liliental-Weber, F. Luckert, R.W. Martin, W. Walukiewicz, C.T. Foxon, J. Appl. Phys. 106 (2009) 103709.

[4] K.M. Yu, S.V. Novikov, R. Broesler, A.X. Levander, Z. Liliental-Weber, F. Luckert, R.W. Martin, O. Dubon, J. Wu, W. Walukiewicz, C.T. Foxon, Phys. Status Solidi C 8 (2011) 2503

[5] R.F. Service, Science 327 (2010) 1598.

[6] F.M. Morales, D. González, J.G. Lozano, R. García, S. Hauguth-Frank, V. Lebedev, V. Cimalla, O. Ambacher, Acta Mater. 57 (2009) 5681.

[7] F.Y. Meng, M. Rao, N. Newman, S. Mahajan, Acta Mater. 56 (2008) 5552.

[8] F.Y. Meng, M. Rao, N. Newman, R. Carpenter, S. Mahajan, Acta Mater. 56 (2008) 4036.

[9] J.G. Lozano, F.M. Morales, R. García, D. González, V. Lebedev, Ch.Y. Wang, V. Cimalla, O. Ambacher, Appl. Phys. Lett. 90 (2007) 091901.

[10] J.L. Hall, A.J. Kent, C.T. Foxon, R.P. Campion, J. Cryst. Growth 312 (2010) 2083.

[11] D. Carvalho, F.M. Morales, Microscopy Microanal., accepted for publication.

[12] A. Putnis, Introduction to Mineral Sciences, Cambridge University Press, Cambridge, 1992.

[13] I. Vurgaftman, J.R. Meyer, L.R. Ram-Mohan, J. Appl. Phys. 89 (2001) 5815.

[14] F.M. Morales, S.I. Molina, D. Araujo, R. García, V. Cimalla, J. Pezoldt, Diamond Rel. Mat. 12 (2003) 1227.

[15] D.M. Tricker, P.D. Brown, T.S. Cheng, C.T. Foxon, C.J. Humphreys, Appl. Surf. Sci. 123 (1998) 22.

[16] S.I. Molina, P.D. Brown, C. Humphreys, J. Cryst. Growth $156(1995) 163$.

[17] F.M. Morales, Ch. Förster, O. Ambacher, J. Pezoldt, Appl. Phys. Lett. 87 (2005) 201910.

[18] T.S. Cheng, L.C. Jenkins, S.E. Hooper, C.T. Foxon, J.W Orton, D.E. Lacklison, Appl. Phys. Lett. 66 (1995) 1509.

[19] S.V. Novikov, C.R. Staddon, C.T. Foxon, F. Luckert, P. Edwards, R. Martin, A.J. Kent, J. Cryst. Growth 323 (2011) 80 . 